\title{
EXPLICIT FORMULAE AND THE LANG-TROTTER CONJECTURE
}

\author{
V. KUMAR MURTY
}

Dedicated to the memories of R. A. Smith and E. G. Straus

1. Introduction Let $E$ be an elliptic curve defined over $\mathbf{Q}$, of conductor $N$. For a prime $p \nmid N$, the reduction $E_{p}$ of $E(\bmod p)$ is an elliptic curve defined over the field $\mathbf{F}_{p}$ of $p$ elements. Denote by $N_{p}$ the number of points of $E_{p}$ which are rational over $\mathbf{F}_{p}$, and write $a_{p}=p+1-N_{p}$. Then it is known that $\left|a_{p}\right| \leqq 2 p^{1 / 2}$. We say that $E$ has super-singular reduction at $p$ if $a_{p}=0$. Define

$$
\pi_{E}(x)=\#\left\{p<x: p \nmid N \text { and } a_{p}=0\right\} .
$$

From results of Deuring [2], it is known that if $E$ has complex multiplication, then, as $x \rightarrow \infty, \pi_{E}(x) \sim(1 / 2) \pi(x)$, where $\pi(x)$ denotes the number of primes $p<x$. If $E$ does not have complex multiplication, Lang and Trotter [4] conjecture that, as $x \rightarrow \infty$,

$$
\pi_{E}(x) \sim C_{E} \frac{x^{1 / 2}}{\log x} .
$$

Serre [6] has shown that, for any $\varepsilon>0$,

$$
\pi_{E}(x) \ll_{\varepsilon} x /(\log x)^{5 / 4-\varepsilon}
$$

and on the assumption of the Riemann Hypothesis for all Artin L-functions, $\pi_{E}(x) \ll x^{3 / 4}$.

For each $p$, write $a_{p}=2 p^{1 / 2} \cos \theta_{p}$ with $\theta_{p} \in[0, \pi]$. Then it is conjectured by Sato and Tate that for any interval $I$ in $(0, \pi)$,

$$
\#\left\{p \leqq x: \theta_{p} \in I\right\} \sim \mu_{E}(I) \pi(x)
$$

for a certain (specified) measure $\mu_{E}$ (cf. [5]). Attached to $E$, there is a family of $\ell$-adic representations

$$
\rho_{\iota}: \operatorname{Gal}(\overline{\mathbf{Q}} / \mathbf{Q}) \rightarrow \mathrm{Gl}_{2}\left(\mathbf{Z}_{\iota}\right)
$$

such that if $p \nmid \ell N$, and $\sigma_{p}$ is a Frobenius element at $p$, then $\rho_{f}\left(\sigma_{p}\right)$ has

Received by the editors November 2, 1983

Copyright (c) 1985 Rocky Mountain Mathematics Consortium 
characteristic polynomial $x^{2}-a_{p} x+p$. We consider the symmetric powers $\operatorname{Sym}^{k} \rho$, of $\rho$, and the $L$-series $L_{k}(s)=L_{k}(s, E)$ attached to them. Each of these series is known to be analytic in a certain half-plane. Suppose we assume the analytic continuation, functional equation, and Riemann Hypotheses for all the $L_{k}$. Let $f(x)$ be any function tending monotonically to $\infty$ with $x$. The main purpose of this paper is to observe that, for any interval $I$ in $(0, \pi)$,

$$
\#\left\{p<x: \theta_{p} \in I\right\}=\mu_{E}(I) \pi(x)+O\left(x^{1 / 2}(\log N x)(\log x) f(x)\right),
$$

provided $x>f^{-1}\left(1 / \mu_{E}(I)\right)$. In particular, this implies that if $E$ does not have complex multiplication,

$$
\pi_{E}(x) \ll x^{3 / 4}(\log N x)^{1 / 2} .
$$

Moreover, the same method shows that, for any integer $a$, and any $E$ defined over $\mathbf{Q}$,

$$
\#\left\{p<x: a_{p}=a\right\} \ll x^{3 / 4}(\log N x)^{1 / 2} .
$$

In the case that $E$ has complex multiplication and $a \neq 0$, this can be improved to $\mathrm{O}\left(x^{1 / 2+\varepsilon}\right)$. Under our hypotheses, it should be possible to prove the estimate $\mathrm{O}\left(x^{1 / 2+\varepsilon}\right)$ in the non complex multiplication case also, but I have not yet been able to do this.

In $\S 2$, we describe the $L_{k}(s)$ and their conjectured functional equations. In $\S 3$, we apply this to derive an explicit formula for $L_{k}(s)$. This is done by the classical method, but the details are included, as it is necessary to keep track of the dependence of various constants on $k$ and other parameters. In $\S 4$, we use the explicit formula to derive an estimate for the sum $\sum_{p<x} F\left(\theta_{p}\right) \log p$, where $\theta_{p} \in[0, \pi]$ and $a_{p}=2 p^{1 / 2} \cos \theta_{p}$, and $F$ is a function on the unit circle satisfying some conditions. Finally, we choose $F$ to approximate the delta function supported at $\theta=\pi / 2$. Using the Riemann Hypothesis, for all $k$, we then deduce the estimate quoted above.

Most of this work was completed in the fall of 1979 while the author was at Harvard University.

2. Symmetric powers and their L-series. There is a strictly compatible rational system $\rho=\left\{\rho_{\ell}\right\}$ of $/$-adic representations

$$
\rho_{\ell}: \operatorname{Gal}(\overline{\mathbf{Q}} / \mathbf{Q}) \rightarrow \mathrm{GL}\left(V_{l}\right), V_{l}=H^{1}\left(E \times_{\mathbf{Q}} \overline{\mathbf{Q}}, \mathbf{Q} /\right) .
$$

The associated $L$-series is of the form

$$
L(s, \rho)=\Pi\left(1-\alpha_{p} p^{-s}\right)^{-1}\left(1-\bar{\alpha}_{p} p^{-s}\right)^{-1},
$$

where, for $p \nmid N,\left|\alpha_{p}\right|=p^{1 / 2}$ and $a_{p}=\alpha_{p}+\bar{\alpha}_{p}$. The $L$-series naturally attached to the system $\operatorname{Sym}^{k} \rho=\left\{\operatorname{Sym}^{k} \rho_{\ell}\right\}$ is 


$$
L_{k}(s)=\prod_{p} \prod_{n=0}^{k}\left(1-\alpha_{p}^{n} \bar{\alpha}_{p}^{k-n} p^{-s}\right)^{-1} .
$$

This product converges absolutely for $\operatorname{Re}(s)>1+(k / 2)$. It is conjectured that $L_{k}(s)$ has an analytic continuation for all $s$ and that it satisfies a functional equation of the following form. Set

$$
\begin{aligned}
& \Gamma_{\mathrm{R}}(s)=\pi^{-s / 2} \Gamma(s / 2) \\
& \Gamma_{\mathrm{C}}(s)=(2 \pi)^{-s} \Gamma(s)
\end{aligned}
$$

and, for each positive integer $k$,

$$
\begin{gathered}
\gamma_{k}(s)=\left\{\begin{array}{c}
\Gamma_{\mathrm{R}}\left(s-\frac{1}{2} k\right) \text { if } k \equiv 0(4) \\
\Gamma_{\mathrm{R}}\left(s-\frac{1}{2} k+1\right) \\
1 \quad \text { if } k \equiv 2(4)
\end{array}\right. \\
\Gamma_{k}(s)=\Gamma_{\mathrm{C}}(s) \Gamma_{\mathrm{C}}(s-1) \cdots \Gamma_{\mathrm{C}}\left(s-\left[\frac{k-1}{2}\right]\right) \gamma_{k}(s) .
\end{gathered}
$$

Thus, for example, $\Gamma_{1}(s)=\Gamma_{\mathrm{C}}(s), \Gamma_{2}(s)=\Gamma_{\mathrm{c}}(s) \Gamma_{\mathrm{R}}(s), \Gamma_{3}(s)=\Gamma_{\mathrm{C}}(s)$ $\Gamma_{\mathrm{c}}(s-1)$, and so on. Now, set

$$
\Lambda_{k}(s)=A_{k}^{s / 2} \Gamma_{k}(s) L_{k}(s),
$$

with a constant $A_{k}$ specified in Serre [7, pp 19-04 to 19-06]. For our purpose, it suffices to note that $A_{1}=N$ and that the following estimate (which follows easily from the definition) holds.

LEMMA 2.1. $A_{k} \leqq N^{k-1}$, for all $k>1$.

Then it is conjectured (cf. Serre [7]) that

$$
\Lambda_{k}(s)= \pm \Lambda_{k}(k+1-s) .
$$

The precise determination of the sign has also been conjectured (by Deligne).

3. Explicit formula. We derive an explicit formula in slightly greater generality than we shall actually need here. Let $K$ be a number field, and set $n_{K}=[K: Q]$. We consider a Dirichlet series

$$
L(s)=\sum_{\mathfrak{a}} c(\mathfrak{a})(N \mathfrak{a})^{-s}=\prod_{v} P_{v}\left((N v)^{-s}\right)^{-1},
$$

where the sum runs over the integral ideals $\mathfrak{a}$ of $K, N$ denotes the norm from $K$ to $\mathbf{Q}$, the product runs over the finite places $v$ of $K$, and 


$$
P_{v}(T)=\prod_{i}\left(1-w_{i, v} T^{i}\right)
$$

is a polynomial in $T$ with real coefficients, $w_{i, v} \in \mathbf{C}$ and $\left|w_{i, v}\right|=1$. We assume that for almost all (i.e., for all but finitely many) $v, \operatorname{deg} P_{v}$ is constant. Let us call it $d$.

Set

$$
\Gamma(s)=\prod_{\lambda} \Gamma\left(\frac{n_{\lambda} s+f_{\lambda}}{2}\right)
$$

where $\lambda$ runs over some finite indexing set, $f_{\lambda}$ are non-negative integers, $n_{\lambda}=1$ or 2 . Let $\gamma_{L}$ denote the number of $\Gamma$ factors. Let $A$ be a real number and set $\Lambda(s)=A^{s} \Gamma(s) L(s)$.

We assume that $\Lambda$ has an analytic continuation to the whole complex plane, except possibly for poles of order $r$ (say) at $s=0$ and 1 , that the continued function is of order 1 , and that there is a functional equation $\Lambda(s)=w \Lambda(1-s)$, with $w= \pm 1$. Under these assumptions, we shall derive an explicit formula for $L(s)$. We shall make use of the approach of Lagarias and Odlyzko [3].

In the calculations below, all implied constants are absolute.

3.1. The first reduction. Let $c>1, x>0$ and $1<T<x$, and consider the integral

$$
I=I_{T}(X)=-\frac{1}{2 \pi i} \int_{c-i T}^{c+i T} \frac{L^{\prime}}{L}(s) x^{s} \frac{d s}{s}
$$

and let

$$
\Omega\left(v^{n}\right)=\sum_{i} w_{i, v}^{n}
$$

Using the discontinuous integral (cf. Davenport [1, p.109])

$$
\frac{1}{2 \pi i} \int_{c-i T}^{c+i T} y^{s} \frac{d s}{s}= \begin{cases}1+O\left(\left(\frac{y}{T}\right)^{c} \min \left(1, \frac{1}{T|\log y|}\right)\right), & \text { if } y>1 \\ O\left(\left(\frac{y}{T}\right)^{c} \min \left(1, \frac{1}{T|\log y|}\right)\right), & \text { if } 0 \leqq y<1 \\ \frac{1}{2}+O\left(\frac{c}{T}\right), & \text { if } y=1,\end{cases}
$$

we find that

$$
\begin{aligned}
I= & \sum_{(N v)^{n}<x} \Omega\left(v^{n}\right) \log (N v)+O\left(\sum_{(N v)^{n}=x} \Omega\left(v^{n}\right) \log (N v)\left(\frac{1}{2}+\frac{c}{T}\right)\right) \\
& +O\left(\sum_{(N v)^{n} \neq x} \Omega\left(v^{n}\right) \log (N v)\left(\frac{x}{T(N v)^{n}}\right)^{c} \min \left(1, \frac{1}{T\left|\log \frac{x}{(N v)^{n}}\right|}\right)\right)
\end{aligned}
$$


As there are at most $n_{K}$ primes above a given rational prime, the first O-term is bounded by $\mathrm{O}\left(d n_{K}(\log x)((1 / 2)+(c / T))\right)$. The sum $\Sigma$ in the second O-term is estimated by splitting it into 3 sums.

$$
\Sigma=\sum_{\mid(N v)^{n-x \mid \leq 1}}+\sum_{\mid(N v)^{n-x \mid \geq x / 4}}+\sum_{1<\mid(N v)^{n-x \mid<x / 4}}=\sum_{1}+\Sigma_{2}+\sum_{3}
$$

Now,

$$
\begin{aligned}
\sum_{1} & \ll \frac{d n_{K} \log x}{T^{c}} \\
\sum_{2} & \ll\left(d \sum_{v, n} \frac{\log (N v)}{(N v)^{n c}}\right)\left(\frac{x}{T}\right)^{c} \\
& \ll d\left(\frac{x}{T}\right)^{c}\left(-\frac{\zeta_{K}^{\prime}}{\zeta_{K}}(c)\right) \ll d n_{K}\left(\frac{x}{T}\right)^{c} \frac{1}{c-1},
\end{aligned}
$$

and

$$
\begin{aligned}
\sum_{3} & \ll \frac{d}{T^{c}} \sum\left(\frac{x}{(N v)^{n}}\right)^{c} \cdot \frac{\log (N v)}{T\left|\log x /(N v)^{n}\right|} \\
& \ll \frac{d(\log x)}{T^{1+c}} \sum_{1<\mid(N v)^{n-x \mid<1 / 4 x}} \frac{1}{\log \left(x /(N v)^{n}\right) \mid} .
\end{aligned}
$$

Since we have the inequalities

$$
|\log y|\left\{\begin{array}{l}
>y-1 \text { for } 0<y<1 \\
>1-\frac{1}{y} \text { for } y>1
\end{array},\right.
$$

the above sum is

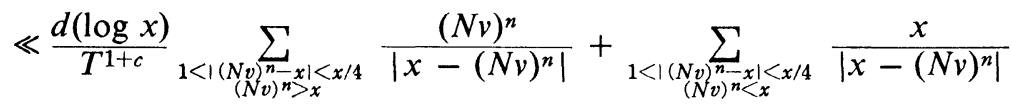

$$
\begin{aligned}
& \ll \frac{d x \log x}{T^{1+c}} n_{K} \sum_{1 \leqq k \leq x / 4} \frac{1}{k} \ll \frac{d n_{K} x(\log x)^{2}}{T^{1+c}} .
\end{aligned}
$$

Putting all this together, we see that if we choose $c=1+(1 / \log x)$, then

$$
\sum_{(N v) n_{<x}} \Omega\left(v^{n}\right) \log (N v)=-\frac{1}{2 \pi i} \int_{c-i T}^{c+i T} \frac{L^{\prime}}{L}(s) \frac{x^{s}}{s} d s+R_{1}(x, T, K, L),
$$

with

$$
R_{1}(x, T, K, L) \ll n_{K} d x\left(\frac{\log x}{T}\right) .
$$

3.2. The behaviour of $L^{\prime} / L$. Now we begin the estimation of the contour integral. Let $j$ be a large positive integer and let $U=(1 / 4)+j$. Write 


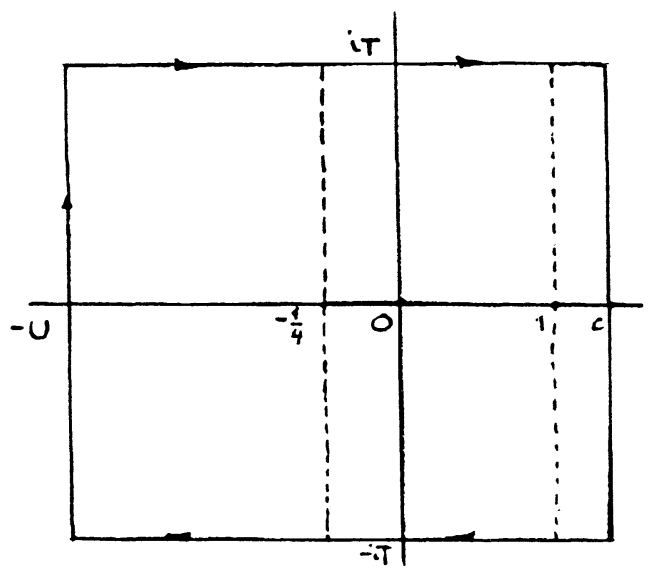

$$
-\frac{1}{2 \pi i} \int_{c-i T}^{c+i T} \frac{L^{\prime}}{L}(s) \frac{x^{s}}{s} d s=-\left(I_{1}+I_{2}+I_{3}+I_{4}+I_{5}\right)+S,
$$

where $I_{1}, \ldots, I_{5}$ are integrals along the indicated segments:

$I_{1}$ is on $-(1 / 4)<\sigma \leqq c, t=-T$;

$I_{2}$ is on $-U \leqq \sigma \leqq-1 / 4, t=-T$;

$I_{3}$ is on $\sigma=-U$. $|t| \leqq T$;

$I_{4}$ is on $-U \leqq \sigma \leqq-1 / 4, t=T$;

$I_{5}$ is on $-(1 / 4)<\sigma \leqq c, t=T$, with $T$ chosen so that $L(\sigma \pm i T) \neq 0$ for any $\sigma$, and $S$ denotes the sum of the residues at poles of the integrand inside this contour. To estimate each of these integrals, we need to know something about the size of $L^{\prime} / L$. This information will be obtained through five lemmas.

LEMMA 1. If $|z+m| \geqq 1 / 8$, for all non-negative integers $m$, then

$$
\left|\frac{\Gamma^{\prime}}{\Gamma}(z)\right| \ll \log (|z|+2) .
$$

This is Lemma 6.1 of [3].

LeMmA 2. If $s=\sigma+\mathrm{it}, \sigma \leqq-1 / 4$ and

$$
\left|n_{\lambda} s+m\right| \geqq \frac{1}{4}
$$

for all non-negative integers $m$, and all $\lambda$, then

$$
\begin{aligned}
\frac{L^{\prime}}{L}(s) \ll \log A+d n_{K} & +\sum_{\lambda}\left\{\log \left(\left|n_{\lambda} s+f_{\lambda}\right|+4\right)\right. \\
& \left.+\log \left(\left|n_{\lambda}(1-s)+f_{\lambda}\right|+4\right)\right\} .
\end{aligned}
$$


Proof. Logarithmically differentiating the functional equation, we obtain

$$
\frac{L^{\prime}}{L}(s)=-\left\{\frac{L^{\prime}}{L}(1-s)+2 \log A+\frac{\Gamma^{\prime}}{\Gamma}(s)+\frac{\Gamma^{\prime}}{\Gamma}(1-s)\right\} .
$$

For $\sigma \leqq-1 / 4, \operatorname{Re}(1-s) \geqq 5 / 4$ and so $\left(L^{\prime} / L\right)(1-s) \ll d n_{K}$. Also, $\left(\Gamma^{\prime} / \Gamma\right)(1-s)$ can be estimated by Lemma 1 . Our hypotheses on $s$ insure that Lemma 1 can be applied to $\left(\Gamma^{\prime} / \Gamma\right)(s)$ also. Since each $n_{\lambda}=1$ or 2 , we have

$$
\frac{\Gamma^{\prime}}{\Gamma}(s)=\sum_{\lambda} \frac{1}{2} n_{\lambda} \frac{\Gamma^{\prime}}{\Gamma}\left(\frac{1}{2}\left(n_{\lambda} s+f_{\lambda}\right)\right) \ll \sum_{\lambda} \log \left(\left|n_{\lambda} s+f_{\lambda}\right|+4\right),
$$

and the lemma follows.

LEMMA 3. For any $s \neq 0,1$ or a zero of $\Lambda(s)$, we have

$$
\operatorname{Re} \frac{\Lambda^{\prime}}{\Lambda}(s)=\sum_{o} \operatorname{Re}\left(\frac{1}{s-\rho}\right)-r \operatorname{Re}\left(\frac{1}{s}+\frac{1}{s-1}\right),
$$

where $r$ is the order of pole of $\Lambda(s)$ at $s=0$, and the sum runs over zeroes $\rho$ of $\Lambda(s)$.

Proof. By the functional equation, the order of pole of $\Lambda(s)$ at $s=0$ and 1 is the same. Thus $[s(s-1)]^{r} \Lambda(s)$ is an entire function of order 1 , and so we have

$$
\Lambda(s)=[s(s-1)]^{-r} a e^{b s} \prod_{\rho}\left(1-\frac{s}{\rho}\right) e^{s / \rho},
$$

where the product is over all zeroes $\rho$ of $\Lambda(s)$. (Note that, for any such $\rho$, we have $0 \leqq \operatorname{Re} \rho \leqq 1)$. We take here the convention that if $s=0$ is a zero of $\Lambda(s)$, then the corresponding factor should be $s$. In particular, for any $s_{1}, s_{2}$ which are not 0,1 or a zero of $\Lambda(s)$, we have

$$
\begin{aligned}
\frac{\Lambda^{\prime}}{\Lambda}\left(s_{1}\right)-\frac{\Lambda^{\prime}}{\Lambda}\left(s_{2}\right)= & \sum_{\rho}\left(\frac{1}{s_{1}-\rho}-\frac{1}{s_{2}-\rho}\right) \\
& -r\left[\frac{1}{s_{1}}+\frac{1}{s_{1}-1}-\frac{1}{s_{2}}-\frac{1}{s_{2}-1}\right] .
\end{aligned}
$$

But, from the functional equation, we see that

$$
\frac{\Lambda^{\prime}}{\Lambda}(1-s)=-\frac{\Lambda^{\prime}}{\Lambda}(s)
$$

and so, in particular,

$$
\frac{\Lambda^{\prime}}{\Lambda}(1-\bar{s})=-\frac{\overline{\Lambda^{\prime}}(s)}{\Lambda}
$$


Therefore, we find that, from (2) and (3),

$$
\begin{aligned}
-2 \operatorname{Re} \frac{\Lambda^{\prime}}{\Lambda}(s)= & \sum\left(\frac{1}{1-\bar{s}-\rho}-\frac{1}{s-\rho}\right) \\
& -r\left[\frac{1}{1-\bar{s}}-\frac{1}{\bar{s}}-\frac{1}{s}-\frac{1}{s-1}\right] .
\end{aligned}
$$

Now, if $\rho$ is a zero of $A(s)$, then so is $1-\bar{\rho}$ (using (2)). Hence, a typical term of the first sum is

$$
\frac{1}{\bar{\rho}-\bar{s}}-\frac{1}{s-\rho}=-2 \operatorname{Re}\left(\frac{1}{s-\rho}\right)
$$

Putting all this together, we deduce that

$$
\operatorname{Re} \frac{\Lambda^{\prime}}{\Lambda}(s)=\sum_{\rho} \operatorname{Re}\left(\frac{1}{s-\rho}\right)-r \operatorname{Re}\left[\frac{1}{s}+\frac{1}{s-1}\right] .
$$

This proves the lemma.

Lemma 4. Let $N(t)$ denote the number of zeroes $\rho=\beta+i \gamma, 0<\beta<1$, $|\gamma-t| \leqq 1$ of $L(s)$. Then

$$
N(t) \ll \log A+d n_{K}+\sum_{\lambda} \log \left(n_{\lambda}|t|+f_{\lambda}+4\right) .
$$

Proof. Let $s=2+$ it. Then, by Lemma 3,

$$
\operatorname{Re} \frac{\Lambda^{\prime}}{\Lambda}(s) \gg \sum_{\mid \gamma-t 1 \leqq 1} \frac{2-\beta}{(2-\beta)^{2}+(t-\gamma)^{2}} \gg N(t)
$$

On the other hand, from Lemma 1,

$$
\left|\frac{\Lambda^{\prime}}{\Lambda}(s)\right| \ll \log A+d n_{K}+\sum_{\lambda} \log \left(n_{\lambda}|t|+f_{\lambda}+4\right),
$$

Since

$$
\operatorname{Re}\left(\frac{\Lambda^{\prime}}{\Lambda}(s)\right) \ll\left|\frac{\Lambda^{\prime}}{\Lambda}(s)\right|
$$

the result follows.

LEMMA 5. If $s=\sigma+i t,-1 / 2 \leqq \sigma \leqq 3,\left|n_{\lambda} s+k\right|>1 / 4$ for all nonnegative integers $k$ and all $\lambda$, and $|s| \geqq 1 / 4$, then

$$
\left|\frac{L^{\prime}}{L}(s)+\frac{r}{s-1}-\sum_{|\lambda-t| \leqq 1} \frac{1}{s-\rho}\right| \ll \log A+d n_{K}+\sum_{\lambda} \log \left(n_{\lambda}|t|+f_{\lambda}+5\right)+r .
$$

Proof. From the formula (1), we see that 


$$
\begin{aligned}
\frac{L^{\prime}}{L}(s) & -\frac{L^{\prime}}{L}(3+i t)=\sum_{\rho}\left(\frac{1}{s-\rho}-\frac{1}{3+i t-\rho}\right) \\
& -\frac{\Gamma^{\prime}}{\Gamma}(s)+\frac{\Gamma^{\prime}}{\Gamma}(3+i t)-r\left(\frac{1}{s}+\frac{1}{s-1}-\frac{1}{3+i t}-\frac{1}{2+i t}\right) .
\end{aligned}
$$

As usual, $L^{\prime} / L(3+\mathrm{it}) \ll d n_{K}$ and the conditions on $s$ imply that Lemma 1 is applicable to $\Gamma^{\prime} / \Gamma(s)$. Hence,

$$
\left|-\frac{\Gamma^{\prime}}{\Gamma}(s)+\frac{\Gamma^{\prime}}{\Gamma}(3+i t)\right| \ll \sum_{\lambda} \log \left(n_{\lambda}|t|+f_{\lambda}+5\right) .
$$

It follows from (4) that

$$
\begin{aligned}
\mid \frac{L^{\prime}}{L}(s) & +\frac{r}{s-1}-\sum_{|r-t| \leq 1}(s-\rho)^{-1} \mid \ll r+\sum_{\lambda} \log \left(n_{\lambda}|t|+f_{\lambda}+5\right) \\
& +\sum_{|r-t|>1}\left|\frac{1}{s-\rho}-\frac{1}{3+i t-\rho}\right|+\sum_{|r-t| \leq 1}|3+i t-\rho|^{-1} .
\end{aligned}
$$

Since $|3+i t-\rho|>1$, the last sum is bounded by $N(t)$. Also,

$$
\begin{aligned}
\sum_{|r-t|>1}\left|\frac{1}{s-\rho}-\frac{1}{3+i t-\rho}\right| & =\sum_{|r-t|>1} \frac{3-\sigma}{|s-\rho||3+i t-\rho|} \\
& \ll \sum_{j \geqq 1} \sum_{j \leq|t-r|<j+1} \frac{3-\sigma}{j^{2}} \\
& \ll \sum_{j \geqq 1} \frac{1}{j^{2}}(N(t+j)+N(t-j)) .
\end{aligned}
$$

Now using Lemma 4, all these estimates together yield

$$
\left|\frac{L^{\prime}}{L}(s)+\frac{r}{s-1}-\sum_{|\gamma-t| \leqslant 1}(s-\rho)^{-1}\right| \ll \log A+d n_{K}+r+\sum_{\lambda} \log \left(n_{\lambda}|t|+f_{\lambda}+5\right) .
$$

3.3. Estimation of the integrals. The lemmas of the previous section immediately yield estimates for $I_{1}, \ldots, I_{5}$. Indeed, since the contour over which $I_{3}$ is taken lies in a region where $s$ satisfies the conditions of Lemma 2 , we deduce that

$$
I_{3} \ll \frac{x^{-U}}{U} T\left(\log A+d n_{K}+\sum_{\lambda} \log \left(n_{\lambda}(T+U)+f_{\lambda}+4\right)\right) .
$$

As our earlier assumption that $T>1$ is still in force, Lemma 2 applies to $I_{2}$ and $I_{4}$ also, so that

$$
I_{2}+I_{4} \ll \frac{x^{-1 / 4}}{T}\left(\log A+d n_{K}+\sum_{\lambda} \log \left(n_{\lambda} T+f_{\lambda}+4\right)\right) .
$$

To estimate $I_{1}$, and $I_{5}$, we use Lemma 5. Indeed, 


$$
\begin{aligned}
I_{1}+I_{5} & =\int_{-1 / 4}^{c}\left(\frac{L^{\prime}}{L}(\sigma+i T) \frac{x^{\sigma+i T}}{\sigma+i T}-\frac{L^{\prime}}{L}(\sigma-i T) \frac{x^{\sigma-i T}}{\sigma-i T}\right) d \sigma \\
& =J_{1}+O\left(J_{2}\right) \text { (say), }
\end{aligned}
$$

where

$$
\begin{aligned}
J_{1}= & \left.\int_{-1 / 4}^{c}\left(\sum_{|\gamma-T| \leq 1} \frac{1}{\sigma+i T-\rho}-\frac{r}{\sigma+i T-1}\right) \frac{x^{\sigma+i T}}{\sigma+i T}\right) d \sigma \\
& -\int_{-1 / 4}^{c}\left(\sum_{|\gamma+T| \leq 1} \frac{1}{\sigma-i T-\rho}-\frac{r}{\sigma-i T-1}\right) \frac{x^{\sigma-i T}}{\sigma-i T} d \sigma
\end{aligned}
$$

and

$$
J_{2} \ll \frac{x}{T \log x}\left\{\log A+d n_{K}+r+\sum_{\lambda} \log \left(n_{\lambda} T+f_{\lambda}+5\right)\right\} .
$$

To estimate $J_{1}$, we use [3, Lemma 6.3$]$ which states

$$
\int_{-1 / 4}^{\alpha} \frac{x^{\sigma+i t}}{(\sigma+i t)(\sigma+i t-\rho)} d \sigma \ll \frac{1}{|t|} x^{\alpha} \frac{1}{\alpha-\beta},
$$

where $\rho=\beta+i_{\gamma}, 0<\beta<1, \gamma \neq t,|t| \geqq 2, x \geqq 2$, and $1<\alpha \leqq 3$. In our case, we use it to deduce that, for $T \geqq 2$,

$$
\begin{aligned}
J_{1} & \ll \frac{x r}{T^{2}}+(N(T)+N(-T)) \frac{x^{c} \log x}{T} \\
& \ll \frac{x r}{T^{2}}+\frac{x \log x}{T}\left(\log A+d n_{K}+\sum_{\lambda} \log \left(n_{\lambda} T+f_{\lambda}+5\right)\right) .
\end{aligned}
$$

Summarizing all of these estimates, we have proved the following

Proposition. Suppose that $2 \leqq T<x$ and $T$ is not the ordinate of a zero of $L(s)$. Then

$$
-\frac{1}{2 \pi i} \int_{c-i T}^{c+i T} \frac{L^{\prime}}{L}(s) x^{s} \frac{d s}{s}=S+R_{2}(x, T, U, L, K)
$$

where $S$ is the sum of the residues from poles of the integrand lying in the rectangle $|\operatorname{Im} s|<T,-U<\operatorname{Re} s<c$, and

$$
\begin{aligned}
R_{2} & \ll \frac{x \log x}{T} \log \left[A e^{d n_{K}} \prod_{\lambda}\left(n_{\lambda} T+f_{\lambda}+5\right)\right] \\
& +\frac{x^{-U}}{U} T \log \left[A e^{d n_{K}} \prod_{\lambda}\left(n_{\lambda}(T+U)+f_{\lambda}+5\right)\right] \\
& +\frac{x r}{T^{2}}+\frac{x r}{T \log x} .
\end{aligned}
$$

3.4. Computation of residues. The poles of $\left(L^{\prime} / L\right)(s) x^{s} / s$ in the rectangle $-U<\sigma<c,|t|<T$ are described as follows. 
(a) Poles coming from zeroes of $L(s)$ in $0<\sigma<1$. These contribute a sum

$$
-\sum_{|r|<T} \frac{x^{\rho}}{\rho}
$$

(b) Poles coming from the trivial zeroes (possibly excluding 0 ). These contribute a sum

$$
-\sum_{\lambda} n_{\lambda} \sum \frac{x^{-\left(2 m+f_{\lambda}\right) / n_{\lambda}}}{2 m+f_{\lambda}}
$$

where the inner sum is over integers $m \geqq 0$, with $0<\left(2 m+f_{\lambda}\right) / n_{\lambda}<U$. Indeed, the functional equation

$$
A^{s} \prod_{\lambda} \Gamma\left(\frac{n_{\lambda} s+f_{\lambda}}{2}\right) L(s)= \pm A^{1-s} \prod_{\lambda} \Gamma\left(\frac{n_{\lambda}(1-s)+f_{\lambda}}{2}\right) L(1-s)
$$

shows that for $s$ to be a trivial zero, we must have $\sigma=\operatorname{Re}(s)<0$ and, for some $\lambda, n_{\lambda} s+f_{\lambda}=-2 m, m$ a non-negative integer. This means that $\sigma=-\left(2 m+f_{\lambda}\right) / n_{\lambda}$ and $t=0$.

(c) Pole of $L(s)$ at $s=1$. This contributes a term $r x$.

(d) A possible (double) pole at $s=0$. The contribution may be calculated as follows. Let $\alpha$ be the number of $\lambda$ such that $f_{\lambda}=0$. Then

$$
\frac{L^{\prime}}{L}(s) \frac{x^{s}}{s}=\left(\frac{\alpha-r}{s}+c_{1}+\text { higher terms }\right)\left(\frac{1}{s}+\log x+\text { higherterms }\right),
$$

for some constant $c_{1}$. Hence

$$
\underset{s=0}{\operatorname{res}}\left(\frac{L^{\prime}}{L}(s) \frac{x^{s}}{s}\right)=(\alpha-r) \log x+c_{1} .
$$

The constant $c_{1}$ can be given in a more useful form. We have

$$
c_{1}=b+r-\log A-\sum_{\lambda: f_{\lambda} \neq 0} \frac{1}{2} n_{\lambda} \frac{\Gamma^{\prime}}{\Gamma}\left(\frac{f_{\lambda}}{2}\right)-\sum_{\lambda: f_{\lambda}=0} \frac{1}{2} n_{\lambda},
$$

where $b$ is the constant in the product (1). Starting from (4), with $3+i t$ replaced by 3 , we find that

$$
\begin{aligned}
\frac{L^{\prime}}{L}(s)=\frac{L^{\prime}}{L}(3) & +\sum_{\rho}\left(\frac{1}{s-\rho}-\frac{1}{3-\rho}\right)-\frac{\Gamma^{\prime}}{\Gamma}(s)+\frac{\Gamma^{\prime}}{\Gamma} \\
& -r\left\{\frac{1}{s}+\frac{1}{s-1}-\frac{5}{6}\right\} .
\end{aligned}
$$

Hence 


$$
\begin{aligned}
c_{1}= & -\sum_{\rho}\left(\frac{1}{\rho}+\frac{1}{3-\rho}\right)+\sum_{\lambda} \frac{1}{2} n_{\lambda} \frac{\Gamma^{\prime}}{\Gamma}\left(\frac{3 n_{\lambda}+f_{\lambda}}{2}\right) \\
& -\sum_{f_{\lambda} \neq 0} \frac{1}{2} n_{\lambda} \frac{\Gamma^{\prime}}{\Gamma}\left(\frac{1}{2} f_{\lambda}\right)-\sum_{f_{\lambda}=0} \frac{1}{2} n_{\lambda}+O\left(d n_{K}\right)+\frac{11}{6} r .
\end{aligned}
$$

As in the proof of Lemma 5, we see that

$$
\sum_{\rho}\left(\frac{1}{\rho}+\frac{1}{3-\rho}\right)=\sum_{|\rho|<1 / 2} \frac{1}{\rho}+O\left(\log \left(A e^{d n_{K}+r} \prod_{\lambda}\left(4+n_{\lambda}+f_{\lambda}\right)\right)\right) .
$$

Indeed, by Lemma 4,

$$
\sum_{|\rho|<1 / 2}\left|\frac{1}{3-\rho}\right| \leqq N(0) \ll \log \left(A e^{d n_{K}} \prod_{\lambda}\left(4+f_{\lambda}\right)\right) .
$$

Also,

$$
\sum_{|\rho| \geqq 1 / 2}\left|\frac{1}{\rho}+\frac{1}{3-\rho}\right| \leqq \sum_{|\rho| \geqq 1 / 2} \frac{3}{|\rho|^{2}} \leqq 3 \sum_{j=1}^{\infty}\left(\frac{N(j)+N(-j)}{j^{2}}\right)+3 \sum_{1 / 2 \leqq 1 \rho \mid \leqq 1} \frac{1}{|\rho|^{2}} .
$$

Using Lemma 4 again,

$$
\begin{aligned}
\sum_{j=1}^{\infty} \frac{N(j)}{j^{2}} & \ll \sum_{j=1}^{\infty} \frac{1}{j^{2}} \log \left[A e^{d n_{K}} \prod_{\lambda}\left(4+n_{\lambda} j+f_{\lambda}\right)\right] \\
& \ll \log A+d n_{K}+\sum_{\lambda} \log \left(4+n_{\lambda}+f_{\lambda}\right),
\end{aligned}
$$

and the other sums are handled similarly. Putting (6) and (7) together, we deduce that

$$
\begin{gathered}
c_{1}=-\sum_{|\rho|<1 / 2} \frac{1}{\rho}-\sum_{f_{\lambda} \neq 0} \frac{1}{2} n_{\lambda} \frac{\Gamma^{\prime}}{\Gamma}\left(\frac{1}{2} f_{\lambda}\right)+O\left(d n_{K}\right)+\left(\frac{11}{6} r\right) \\
+O\left(\log \left[A e^{d n_{K}+r} \prod_{\lambda}\left(4+n_{\lambda}+f_{\lambda}\right)\right]\right) .
\end{gathered}
$$

REMARK. The method of this section, which is essentially based on [3] can also be used in the following more general setting. We drop the condition that the $P_{v}(T)$ have real coefficients and we define

$$
\check{L}(s)=\prod_{v} \prod_{i}\left(1-\bar{w}_{i, v}(N v)^{-s}\right)^{-1} .
$$

For each $\lambda$, let $\varphi_{\lambda}$ be some real number, and set $s_{\lambda}=s+i \varphi_{\lambda}, s_{\bar{\lambda}}=$ $s-i \varphi_{\lambda}$. Define

$$
\Gamma(s)=\prod_{\lambda} \Gamma\left(\frac{n_{\lambda} s_{\lambda}+f_{\lambda}}{2}\right)
$$

and

$$
\check{\Gamma}(s)=\prod_{\lambda} \Gamma\left(\frac{n_{\lambda} s_{\bar{\lambda}}+f_{\lambda}}{2}\right) .
$$


Set $\Lambda(s)=A^{s} \Gamma(s) L(s), \check{\Lambda}(s)=A^{s} \breve{\Gamma}(s) \breve{L}(s)$ and suppose that $\Lambda$ and $\breve{\Lambda}$ have an analytic continuation and satisfy the functional equation

$$
\Lambda(s)=\omega \check{\Lambda}(1-s)
$$

with $\omega \in \mathbf{C},|\omega|=1$. In this case the Proposition of 3.3 in valid for $\max \left(2,1 / 4+\max _{\lambda} \varphi_{\lambda}\right) \leqq T<x$, provided we replacd the term $\log \left(n_{\lambda} T+f_{\lambda}+5\right)$ in $R_{2}$ by

$$
\log \left(n_{\lambda}\left|T+\varphi_{\lambda}\right|+f_{\lambda}+5\right)+\log \left(n_{\lambda}\left|T-\varphi_{\lambda}\right|+f_{\lambda}+5\right)
$$

In the calculation of $S$, the contribution of (b) in 3.4 has to be replaced by

$$
-\sum_{\lambda} n_{\lambda} \Sigma \frac{\exp \left(-\left(\frac{2 m+f_{\lambda}}{n_{\lambda}}+i \varphi_{\lambda}\right) \log x\right)}{2 m+f_{\lambda}+i \varphi_{\lambda} n_{\lambda}},
$$

and in the contribution of (d), $\alpha$ is the number of $\lambda$ such that

$$
\varphi_{\lambda}=0 \text { and } f_{\lambda}=0,
$$

and

$$
\begin{aligned}
c= & -\sum_{|\rho|<1 / 2} \frac{1}{\rho}+O\left(\log \left[A e^{d n_{K}+r} \prod_{\lambda}\left(4+n_{\lambda}\left(1+\left|\varphi_{\lambda}\right|\right)+f_{\lambda}\right)\right]\right) \\
& -\sum_{\varphi_{\lambda} \circ r f_{\lambda} \neq 0} \frac{1}{2} n_{\lambda} \frac{\Gamma^{\prime}}{\Gamma}\left(\frac{f_{\lambda}+i n_{\lambda} \varphi_{\lambda}}{2}\right)+O\left(d n_{K}\right)+O\left(\gamma_{L}\right)+\frac{11}{6} r .
\end{aligned}
$$

4. Application. In this section, we apply the explicit formulae of $\S 3$ to the $L$-series $L_{k}(s)$ of $\S 2$. We shall assume, throughout, that all of the $L_{k}$

(i) have an analytic continuation to the entire $s$-plane as functions of order 1;

(ii) satisfy the conjectured functional equation which was described in $\S 2$.

We shall also assume that $E$ does not have complex multiplication. Write

$$
-\frac{L_{k}^{\prime}}{L_{k}}\left(s+\frac{k}{2}\right)=\sum_{p, n} \Omega_{k}\left(p^{n}\right) p^{-n s} .
$$

Proposition 4.1. Suppose the Riemann Hypothesis is true for $L_{k}$. Then

$$
\sum_{\substack{p \leq x \\ p \nmid N}} \Omega_{k}(p) \log p=\delta_{k} x+O\left(k x^{1 / 2}(\log x) \log (N(x+k))\right),
$$

where $\delta_{k}=1$ if $k=0$ and $\delta_{k}=0$ otherwise.

Proof. The Proposition of $\S 3.3$ gives an expression of the form

$$
\sum_{\substack{p<x \\ p \nmid N}} \Omega_{k}\left(p^{n}\right) \log p=S_{k}(U, T)+R_{k}(U, T)
$$

where $S_{k}(U, T)$ is a sum of residues of $-\left(L_{k}^{\prime} / L_{k}\right)(s+(k / 2))\left(x^{s} / s\right)$ and 
$R_{k}(U, T)$ is an error term. The conditions on $U$ and $T$ are that $2 \leqq T<x$ and $T$ is not the ordinate of a zero of $L_{k}(s)$, and $U=1 / 4+j, j$ a large integer. In this expression, we let $U \rightarrow \infty$. It is clear from $\$ 3.4$ that $S_{k}(U, T)$ converges. Furthermore, by the usual argument [1, p. 114] we may choose any $T$ such that $2 \leqq T<x$. Taking $T=x-1$, we find that

$$
R_{k} \ll k(\log x) \log \left(\left(x+\frac{k}{2}+5\right) A_{1} e\right) .
$$

The assumption (i) implies [5] that $L_{k}(s+k / 2) \neq 0$, for $\operatorname{Re}(s)=1$ and $k>0$. Hence, the order $r_{k}$ of $L_{k}(s+(k / 2))$ at $s=1$ is given by $r_{k}=\delta_{k}$. Furthermore, the sum over trivial zeroes is easily seen to be

$$
\begin{array}{ll}
-\sum_{j=0}^{[k / 2]} 2 \sum_{a=0}^{\infty}(2 a+2 j+1)^{-1} x^{-(a+j+1 / 2)}, & \text { for } k \text { odd, } \\
-\sum_{j=1}^{k / 2} 2 \sum_{a=0}^{\infty}(a+j)^{-1} x^{-(a+j)}-\sum_{a=1}^{\infty}(2 a)^{-1} x^{-2 a}, & \text { for } k \equiv 0(4), \\
-\sum_{j=1}^{k / 2} 2 \sum_{a=0}^{\infty}(a+j)^{-1} x^{-(a+j)}-\sum_{a=0}^{\infty}(2 a+1)^{-1} x^{-(2 a+1)}, \text { for } k \equiv 2(4) .
\end{array}
$$

A straightforward calculation shows that these sums are $O(1 / x)$ uniformly in $k$.

Putting all this together, we find that for $2 \leqq T<x$,

$$
\begin{aligned}
\sum_{\substack{p^{n} \leq x \\
p \times N}} \Omega_{k}\left(p^{n}\right) \log p=\delta_{k} x & +B_{k}-\sum_{\mid \gamma_{k} \leq T} \eta_{k}^{-1} x^{\eta_{k}} \\
& +O\left(k(\log x) \log \left(\left(x+\frac{k}{2}+5\right) A_{1} e\right)\right),
\end{aligned}
$$

where we have used Lemma 2.1 and

$$
B_{k}=-\sum_{\left|\eta_{k}\right|<1 / 2} \frac{1}{\eta_{k}}-\sum_{0 \leqq j<k / 2} \frac{\Gamma^{\prime}}{\Gamma}\left(\frac{k}{2}-j\right)+O\left(k \log \left((k+10) A_{1} e\right)\right)
$$

and $\eta_{k}$ denotes a zero of $L_{k}(s+k / 2)$, and $\gamma_{k}=\operatorname{Im} \eta_{k}$.

First, we observe that

$$
\sum_{\substack{p_{n \leq x} \leq x \\ n=2 \\ p \ngtr N}} \Omega_{k}\left(p^{n}\right) \log p \ll k x^{1 / 2} .
$$

Next, by Lemma 3.1,

$$
\sum_{0 \leqq j<k / 2} \frac{\Gamma^{\prime}}{\Gamma}\left(\frac{k}{2}-j\right) \ll k \log k
$$

Finally, using the Riemann Hypothesis, 


$$
\begin{aligned}
\sum_{|\gamma k| \leqslant T} & \eta_{k}^{-1} x^{\eta_{k}}+\sum_{\left|\eta_{k}\right|<1 / 2} \eta_{k}^{-1} \ll x^{1 / 2} \sum_{\left|\gamma_{k}\right| \leq T}\left|\eta_{k}\right|^{-1} \\
& \ll x^{1 / 2} \sum_{j=1}^{T} \frac{1}{j}\left\{N_{k}(j)+N_{k}(-j)\right\} \\
& \ll x^{1 / 2} \sum_{j=1}^{T} \frac{1}{j} \log \left[\left(A_{1} e\right)^{k+1} \prod_{0 \leq \lambda \leq k / 2}\left(j+\frac{k}{2}-\lambda+2\right)\right] \\
& \ll k x^{1 / 2}(\log T) \log \left(A_{1} e\left(T+\frac{k}{2}+2\right)\right) \\
& \ll k x^{1 / 2}(\log x) \log \left(A_{1} e\left(x+\frac{k}{2}+2\right)\right)
\end{aligned}
$$

by our choice of $T$. Putting all of this together, we find that

$$
\begin{aligned}
\sum_{\substack{p \leq x \\
p \nmid N}} \Omega_{k}(p) \log p & =\delta_{k} x+O\left(k x^{1 / 2}(\log x) \log \left(A_{1} e\left(x+\frac{k}{2}+2\right)\right)\right) \\
& =\delta_{k} x+O\left(k x^{1 / 2}(\log x) \log (N(x+k))\right) .
\end{aligned}
$$

This proves the result.

Choose a real number $\delta$ such that $0<\delta<1 / 4$. By a result of Vinogradov [8, Ch. 1, Lemma 12], there is a periodic function $D(x)$ on $\mathbf{R}$ of period 1 satisfying

(i) $D(x)=1$ on $[(1 / 4)-(1 / 2) \delta,(1 / 4)+(1 / 2) \delta]$;

(ii) $D(x)=0$ on $[(1 / 4)+(3 / 2) \delta,(5 / 4)-(3 / 2) \delta]$;

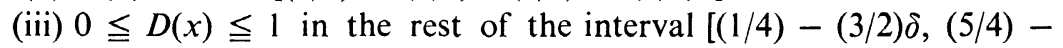
$(3 / 2) \delta]$;

(iv) if we write $D(x)=c_{0}+\sum_{m=1}^{\infty}\left(c_{m} \cos 2 \pi m x+d_{m} \sin 2 \pi m x\right)$ then $c_{0}=2 \delta$, and $\left|c_{m}\right|,\left|d_{m}\right| \ll m^{-2} \delta^{-1}$ for all $m \geqq 1$.

We set $F(\theta)=D(\theta / 2 \pi)+D(-\theta / 2 \pi)$. Then $F$ is an even function of period $2 \pi$ which takes the value 1 on $[(\pi / 2-\pi \delta,(\pi / 2)+\pi \delta] \cup[-(\pi / 2)-$ $\pi \delta,-(\pi / 2)+\pi \delta]$, and if we write

$$
F(\theta)=\sum_{m \Subset \mathbf{Z}} a_{m} e^{i m \theta},
$$

then $\left|a_{0}-a_{2}\right| \ll \delta$.

We have, for any $M>1$,

$$
F(\theta)=\sum_{|n| \leq M} a_{n} e^{i n \theta}+O\left(\delta^{-1} M^{-1}\right) .
$$

Furthermore, setting

$$
\chi_{k}(\theta)=\sum_{j=0}^{k} e^{i \theta(k-2 j)},
$$

we have 
(10) $F(\theta)=\left(a_{0}-a_{2}\right)+2\left(a_{1}-a_{3}\right) \cos \theta+\sum_{n=2}^{M-2}\left(a_{n}-a_{n+2}\right) \chi_{n}(\theta)+O\left(\delta^{-1} M^{-1}\right)$.

For $p \nmid N$, the $p$-th Euler factor of $L_{1}(s+1 / 2)=L(s+1 / 2, \rho)$ is

$$
\left(1-\alpha_{p} p^{-s}\right)^{-1}\left(1-\bar{\alpha}_{p} p^{-s}\right)^{-1},\left|\alpha_{p}\right|=1 \text {. }
$$

Write $\alpha_{p}=e^{i \theta_{p}}$ so that $\theta_{p}$ is determined up to sign. We have

$$
\Omega_{k}(p)=\sum_{j=0}^{k} \alpha_{p}^{j} \bar{\alpha}_{p}^{k-j}=\sum_{j=0}^{k} e^{i \theta_{p}(k-2 j)}=\chi_{k}\left(\theta_{p}\right) .
$$

Substituting into (10), summing over $p$, and observing that

$$
\sum_{\substack{p \leq x \\ p \backslash N}} \log p=\psi(x)+O((\log N)(\log x)),
$$

we have

$$
\begin{aligned}
\sum_{\substack{p \leq x \\
p \nmid N}} F\left(\theta_{p}\right) \log p= & \left(a_{0}-a_{2}\right) \psi(x)+\sum_{k=1}^{M-2}\left(a_{k}-a_{k+2}\right) \sum_{\substack{p \leq x \leq \\
p \nmid N}} \Omega_{k}(p) \log p \\
& +O\left(\left(a_{0}-a_{2}\right)(\log N)(\log x)\right)+O\left(\delta^{-1} M^{-1} \psi(x)\right) .
\end{aligned}
$$

Using the estimate $\left|a_{k}\right| \ll \delta^{-1} k^{-2}$ and Proposition 4.1, we see that the Riemann Hypothesis for all the $L_{k}$ implies that

$$
\sum_{k=1}^{M-2}\left(a_{k}-a_{k+2}\right) \sum_{\substack{p \leq x \\ p \backslash N}} \Omega_{k}(p) \log p \ll \delta^{-1} x^{1 / 2}(\log x) \log (N(x+M)) \log M .
$$

If we choose $M=\delta^{-2}$, we deduce that

$\sum_{\substack{p \leq x \\ p \nmid N}} F\left(\theta_{p}\right) \log p=O(\delta \psi(x))+O\left(\delta^{-1} x^{1 / 2}(\log x)\left(\log \left(N\left(x+\delta^{-2}\right)\right)\right) \log \left(\delta^{-2}\right)\right)$

and also that

$$
\pi_{E}(x) \ll \delta \pi(x)+\delta^{-1} x^{1 / 2}\left(\log N\left(x+\delta^{-2}\right)\right)\left(\log \delta^{-2}\right) .
$$

As the implied constant does not depend on $\delta$, we may choose $\delta=$ $x^{-1 / 4}(\log N x)^{1 / 2} \log x$. This yields the following result.

Proposition 4.2. Suppose the Riemann Hypothesis is true for all the $L_{k}$. Then

$$
\pi_{E}(x) \ll x^{3 / 4}(\log N x)^{1 / 2} .
$$

The more general result stated in the Introduction is proved similarly.

\section{REFERENCES}

1. H. Davenport, Multiplicative Number Theory, Markham, Chicago, 1967.

2. M. Deuring, Die Zetafunktion einer algebraischen Kurve vom Geschlechte Eins, 
Nachr. Akad. Wiss. Gottingen, in four parts (1953) 85-94, (1955) 13-42, (1956) 37-76, (1957) 55-80.

3. J. Lagarias and A. M. Odlyzko, Effective versions of the Chebotarev density theorem, in Algebraic Number Fields, ed. A. Frohlich. Academic Press, New York, 1977, 409464.

4. S. Lang and H. Trotter, Frobenius distributions in $\mathrm{GL}_{2}$ extensions, Lecture Notes in Mathematics 504, Berlin, Springer-Verlag, 1976.

5. V. Kumar Murty, On the Sato-Tate conjecture, in: Number Theory related to Fermat's last theorem, ed. N. Koblitz, Birkhauser-Verlag, Boston, 1982, 195-205.

6. J.-P. Serre, Quelques applications du théorème de densité de Chebotarev, Publ. Math. IHES 54 (1982), 123-201.

7. J.-P. Serre, Facteurs locaux des fonctions zêta des variétés algébriques (Definitions et Conjectures), Sém. Délange-Pisot-Poitou 1969/70, Exposé 19.

8. I. M. Vinogradov, The method of trigonometrical sums in the Theory of numbers, London, Interscience, 1947.

School of Mathematics, Tata Institute of Fundamental Research, Bombay, India 
